PROCEEDING ;S OF THE

AMERICAN MATHEMATICAL SOCIETY

Volume 87. Number 2. February 1983

\title{
ON A CERTAIN CLASS OF $M_{1}$-SPACES
}

\author{
T. MIZOKAMI
}

\begin{abstract}
Let 'y' be the class of all $M_{1}$-spaces whose every closed subset has a closure-preserving open neighborhood base. A characterization is given, and it is proved that the adjunction space $X \cup, Y$ is an $M_{1}$-space if $X \in Y Y$ and $Y$ is an $M_{1}$-space. Moreover, it is proved that if $X$ is a space such that for each metrizable space $Y$. every closed subspace of $X \times Y$ is an $M_{1}$-space, then $X \in$ s.
\end{abstract}

1. Introduction. Let $\left(\mathrm{P}_{1}\right), i=1,2,3,4$, be the following statements concerning $M_{1}$-spaces [3] and stratifiable spaces [1]:

$\left(\mathrm{P}_{1}\right)$ Every stratifiable space is an $M_{1}$-space.

$\left(\mathrm{P}_{2}\right)$ Each closed subspace of an $M_{1}$-space is also an $M_{1}$-space.

$\left(\mathrm{P}_{3}\right)$ Each adjunction space of $M_{1}$-spaces is also an $M_{1}$-space.

$\left(\mathrm{P}_{4}\right)$ Each closed subset of an $M_{1}$-space has a $\sigma$-closure-preserving open neighborhood base.

The problems whether the $\left(\mathrm{P}_{i}\right)$ are true or not are still open. The problems of $\left(\mathrm{P}_{1}\right)$. $\left(\mathrm{P}_{2}\right)$ and $\left(\mathrm{P}_{3}\right)$ are posed by Ceder [3]. On the other hand, the problem of $\left(\mathrm{P}_{4}\right)$ is posed by Borges and Lutzer in [2], where they characterized stratifiable spaces (which coincide with $M_{2}$-spaces by [4 and 8]) as paracompact $\sigma$-spaces such that each closed subset has a $\sigma$-closure-preserving neighborhood base which consists of not necessarily open neighborhoods. By Borges [1] and by the recent result of Heath and Junnila [6], it follows that $\left(\mathrm{P}_{1}\right) \rightleftarrows\left(\mathrm{P}_{2}\right) \rightarrow\left(\mathrm{P}_{3}\right) \rightarrow\left(\mathrm{P}_{4}\right)$. As is well known by Ceder [3, Lemma 7.3], every closed subset of an $M_{2}$-space has a closure-preserving neighborhood base. Corresponding to this, let $\left(\mathrm{P}_{4}\right)^{\prime}$ be the statement as follows:

$\left(\mathrm{P}_{4}\right)^{\prime}$ Every closed subset of an $M_{1}$-space has a closure-preserving open neighborhood base.

Obviously $\left(\mathrm{P}_{4}\right)^{\prime} \rightarrow\left(\mathrm{P}_{4}\right)$. The author in this paper studies the class $\mathscr{P}$ of $M_{1}$-spaces satisfying $\left(\mathrm{P}_{4}\right)^{\prime}$ and proves that if $\left(\mathrm{P}_{2}\right)$ is assumed to be true, then $\left(\mathrm{P}_{4}\right)^{\prime}$ holds.

In this paper, all spaces are assumed to be regular and Hausdorff and all mappings to be continuous and onto unless the contrary is stated explicitly. $N$ always denotes the positive integers. To state the results and the proofs, we give the definitions to the terminology used below. A mapping $f: X \rightarrow Y$ is said to be irreducible if no closed proper subspace of $X$ is mapped onto $Y$ by $f$. A space $X$ is said to be monotonically normal [7] if the following is satisfied: To each pair $(H, K)$

Received by the editors September 21, 1981 and, in revised form, June 14, 1982.

1980 Mathematics Subject Classification. Primary 54E20.

Key words and phrases. Stratifiable, $M_{1}$-space, irreducible mapping, adjunction space, property ECP.

(c) 1983 American Mathematical Society 0002-9939/82/0000-0802/\$02.00 
of separated subsets of $X$ one can assign an open set $D(H, K)$ in such a way that

(i) $H \subset D(H, K) \subset \overline{D(H, K)} \subset X-K$, and

(ii) if $\left(H^{\prime}, K^{\prime}\right)$ is a pair of separated sets having $H \subset H^{\prime}$ and $K^{\prime} \subset K$, then $D(H, K) \subset D\left(H^{\prime}, K^{\prime}\right)$.

According to [7], every stratifiable space is monotonically normal, and this fact is used below.

\section{The class $\odot$.}

Definition 1. Let $\mathcal{P}$ be the class of all $M_{1}$-spaces satisfying $\left(\mathrm{P}_{4}\right)^{\prime}$.

Most common examples of $M_{1}$-spaces seem to belong to $\mathcal{P}$. For example: (1) stratifiable $F_{\sigma}$-metrizable spaces due to Gruenhage [5], (2) $M_{1}$-spaces with dim $\leqslant 0$ and (3) the closed image of an $M_{0}$-space (a space which has a $\sigma$-closure-preserving base consisting of closed and open sets) belong to $\mathcal{P}$. The proofs are given below implicitly. Every Lašnev space, and more generally every L-space of Nagami [9], belongs to $\stackrel{P}{ }$, but the converse is not true as shown by [9, Examples 2.1, 2.2].

LEMMA 1. Let $M$ be a closed subspace of a stratifiable space $X$. Then to each open set $U$ of $M$, we can assign an open set $U^{\prime}$ of $X$ such that $U^{\prime} \cap M=U$ and the following is satisfied:

(*) $U^{\prime} \cap V^{\prime}=\varnothing$ whenever $U, V$ are disjoint open sets of $M$.

Proof. Let $\widetilde{F}=\bigcup\left\{\mathscr{F}_{n}: n \in N\right\}$ be a $\sigma$-locally finite closed network of $M$ such that each $\widetilde{F}_{n}$ is locally finite and $\widetilde{F}_{n} \subset \widetilde{F}_{n+1}$. For each open set $W$ of $M$ and each $n \in N$, set

$$
\widetilde{\mathcal{F}}_{n}(W)=\left\{F \in \widetilde{\mathcal{F}}_{n}: F \subset W\right\}, \quad \mathscr{F}_{n}(W)^{\sharp}=\bigcup\left\{F: F \in \mathscr{F}_{n}(W)\right\} .
$$

We shall construct $U^{\prime}$ for an arbitrary open set $U$ of $M$ by

$$
\begin{gathered}
U_{n}=D\left(\mathscr{\mathscr { F }}_{n}(U)^{\#}, M-U\right)-\overline{D\left(\mathscr{F}_{n}(M-\bar{U})^{\#}, \bar{U}\right)}, \quad n \in N, \\
U^{\prime}=\bigcup\left\{U_{n}: n \in N\right\} .
\end{gathered}
$$

Obviously $U^{\prime} \cap M=U$. To see (*) for disjoint open sets $U, V$ of $M$, assume that $V_{m} \cap U_{n} \neq \varnothing, m, n \in N$. Without loss of generality, we can assume $n \geqslant m$. Observe

$$
D\left(\mathscr{F}_{m}(V)^{\sharp}, M-V\right) \subset D\left(\mathscr{F}_{n}(M-\bar{U})^{\sharp}, \bar{U}\right) .
$$

This contradicts the fact $V_{m} \cap U_{n} \neq \varnothing$.

In the above proof, if we use only the fact that $X$ is monotonically normal, we obtain the following:

COROLlary. Let $M$ be a closed subspace of a monotonically normal space $X$ and let $\left\{U_{\alpha}: \alpha \in A\right\}$ be an open family of $M$. Then there exists an open family $\left\{U_{\alpha}^{\prime}: \alpha \in A\right\}$ of $X$ such that $U_{\alpha}^{\prime} \cap M=U_{\alpha}$ for each $\alpha \in A$ and for each $B \subset A$

$$
\overline{\bigcup\left\{U_{\alpha}: \alpha \in B\right\}}=\overline{\bigcup\left\{U_{\alpha}^{\prime}: \alpha \in B\right\}} \cap M \text {. }
$$

In fact, if we define

$$
U_{\alpha}^{\prime}=\bigcup\left\{D\left(\{x\}, M-U_{\alpha}\right): x \in U_{\alpha}\right\}, \quad \alpha \in A,
$$

then $\left\{U_{\alpha}^{\prime}: \alpha \in A\right\}$ satisfied the required property. 
Definition 2. (Nagata [10, Definition 2]). A space $X$ is said to have the property (ECP) when the following is satisfied: If $X^{\prime}=X \cup F$, where $X, F$ are closed in $X^{\prime}$, and if $\mathcal{Q}=\left\{U_{\alpha}: \alpha \in A\right\}$ is a closure-preserving open collection in the subspace $F$, then for each $\alpha \in A$ there is a family $\left\{U_{\beta}^{\prime}: \beta \in B_{\alpha}\right\}$ of open sets of $X^{\prime}$ satisfying (1) $U^{\prime}=\left\{U_{\beta}^{\prime}: \beta \in \cup\left\{B_{\alpha}: \alpha \in A\right\}\right\}$ is closure-preserving in $X^{\prime}$, (2) for each $\beta \in B_{\alpha}$, $U_{\beta}^{\prime} \cap F=U_{\alpha}$ and for every open set $V$ in $X^{\prime}$ with $V \cap F=U_{\alpha}$, there is $\beta \in B_{\alpha}$ such that $U_{\alpha} \subset U_{\beta}^{\prime} \subset V$, (3) for every open set $W$ of $F$, there is an open set $W^{\prime}$ of $X^{\prime}$ such that $W^{\prime} \cap F=W$ and such that $W^{\prime} \cap U_{\beta}^{\prime}=\varnothing$ whenever $\beta \in B_{\alpha}$ and $W \cap U_{\alpha}=\varnothing$.

THEOREM 1. The following are equivalent for a stratifiable space $X$ :

(1) $X \in \mathscr{P}$.

(2) For each closed subspace $M$ of $X$, there exists an open family $\mathcal{Q}$ of $X$ such that $\mathscr{U}$ is closure-preserving in $X-M$ and for each open set $V$ of $X$ there exists $U \in \mathcal{Q}$ such that $U \subset V$ and $U \cap M=V \cap M$.

(3) $X$ has (ECP).

Proof. (3) $\rightarrow(1)$ is trivial.

(1) $\rightarrow$ (2): Let $\left\{G_{n}: n \in N\right\}$ be a sequence of open sets of $X$ such that $M=$ $\cap\left\{G_{n}: n \in N\right\}, \bar{G}_{n+1} \subset G_{n}, n \in N$. Let $\mathscr{F}=\cup\left\{\mathscr{F}_{n}: n \in N\right\}$ be a closed network for $M$ such that each $\mathscr{F}_{n}$ is discrete. For each $n \in N$, let $\left\{G_{F}: F \in \mathscr{F}_{n}\right\}$ be an open discrete family of $X$ such that $F \subset G_{F}$ for each $F \in \mathscr{F}_{n}$ and $\cup\left\{G_{F}: F \in \mathscr{F}_{n}\right\} \subset G_{n}$. Let $\mathcal{U}_{n}$ be a closure-preserving open neighborhood base for $\cup\left\{F: F \in \mathscr{F}_{n}\right\}$. Set

$$
\mathcal{U}_{n}^{\prime}=\left\{U \cap G_{F}: F \in \mathscr{F}_{n}, U \in \mathcal{U}_{n}, \bar{U} \subset \cup\left\{G_{F}: F \in \mathscr{F}_{n}\right\}\right\} .
$$

Note that $\mathscr{U}_{n}^{\prime}$ is closure-preserving in $X$. For the family $\mathscr{U}$, take the collection consisting of all unions of the sets from $\cup\left\{\mathcal{U}_{n}^{\prime}: n \in N\right\}$. Then it is easily shown that $\mathcal{Q}$ has the desired property.

$(2) \rightarrow(3)$. Let $X^{\prime}=X \cup F$, where $F, X$ are closed in $X^{\prime}$. Suppose $\mathscr{U}=\left\{U_{\alpha}\right.$ : $\alpha \in A\}$ is a closure-preserving open family of the subspace $F$. By (2) there exists an open family $\mathfrak{V}$ of $X$ such that $\mathfrak{V}$ is closure-preserving in $X-F$ and for each open set $W$ of $X$ there exists $V \in \mathscr{V}$ such that $V \subset W$ and $V \cap(F \cap X)=W \cap(F \cap X)$. For each $\alpha \in A$, we construct $\mathscr{V}_{\alpha}$ as follows:

$$
\mathscr{V}_{\alpha}=\left\{V \in \mathscr{V}: V \cap(F \cap X)=U_{\alpha} \cap(F \cap X), V \subset\left(U_{\alpha} \cap F \cap X\right)^{\prime}\right\},
$$

where $\left(U_{\alpha} \cap F \cap X\right)^{\prime}$ is the special extension of $U_{\alpha} \cap F \cap X$ to $X$ assured by Lemma 1. Write $\mathcal{T}_{\alpha}=\left\{V_{\beta}: \beta \in B_{\alpha}\right\}, \alpha \in A$. Set for each $\alpha \in A, U_{\beta}^{\prime}=V_{\beta} \cup U_{\alpha}$, $\beta \in B_{\alpha}$. Then it is easily seen that $\left\{U_{\beta}^{\prime}: \beta \in B_{\alpha}, \alpha \in A\right\}$ satisfies the required conditons. This completes the proof.

Corollary 1. Let $X \in \mathcal{P}$ and let $M$ be a closed subspace of $X$. If $\mathscr{U}$ is $a$ closure-preserving open family of $M$, then there exists a closure-preserving family $U^{\prime}$ of open sets of $X$ such that for every open subset $V$ of $X$ with $V \cap M \in \mathcal{U}$, there exists $U^{\prime} \in U^{\prime}$ such that $U^{\prime} \subset V$ and $U^{\prime} \cap M=V \cap M$.

The following is easily obtained by a repetition of the proof of $[10$, Theorem 1]:

Corollary 2. Let $\left\{X_{i}: i \in N\right\}$ be a closed cover of a space $X$. If each $X_{i} \in \mathcal{P}$ and $\left\{X_{i}\right\}$ dominates $X$, then $X \in \mathcal{O P}$. 
The following Corollary 3 and Theorem 2 are obtained by modifying the proof [10, Lemma 2]. We give the proof only to Theorem 2, and that of Corollary 3 is similar.

COROLlARY 3. The adjunction space of $X, Y \in \mathscr{P}$ belongs to $\mathscr{P}$.

THEOREM 2. Let $X \in \mathcal{P}$ and let $Y$ be an $M_{1}$-space. Then the adjunction space $Z=X \cup_{f} Y$ is an $M_{1}$-space.

Proof. Let $f$ be a mapping of a closed subset $H$ of $X$ into $Y$. Let $p: X \vee Y \rightarrow Z$ be the quotient mapping. Let $\mathscr{U}=\cup\left\{\mathcal{U}_{n}: n \in N\right\}$ be a $\sigma$-closure-preserving base for $p(Y)$, where each $\mathscr{Q}_{n}=\left\{U_{\alpha}: \alpha \in A_{n}\right\}$ is closure-preserving in $p(Y)$. By (2) of Theorem 1, there exists an open family $\mathcal{V}$ of $X$ such that $V$ is closure-preserving in $X-H$ and for each open set $U$ of $X$ there exists $V \in \mathcal{V}$ such that $V \subset U$ and $V \cap H=U \cap H$. Let $n \in N$ be fixed for a while. Set

$$
\begin{gathered}
\mathscr{V}(\alpha)=\left\{V \in \mathcal{T}: V \cap H=p_{X}^{-1}\left(U_{\alpha}\right), V \subset\left(p_{X}^{-1}\left(U_{\alpha}\right)\right)^{\prime}\right\}, \quad \alpha \in A_{n}, \\
\mathscr{U}(\alpha)=\left\{p(V) \cup U_{\alpha}: V \in \mathscr{V}(\alpha)\right\}, \quad \alpha \in A_{n},
\end{gathered}
$$

where $p_{X}=p \mid X$ and $\left(p_{X}^{-1}\left(U_{\alpha}\right)\right)^{\prime}$ is the special extension of $p_{X}^{-1}\left(U_{\alpha}\right)$ to $X$ assured by Lemma 1. Set $\mathscr{U}=\bigcup\left\{\mathcal{U}(\alpha): \alpha \in A_{n}, n \in N\right\}$. Then it is easily seen that $\mathscr{W}$ is a $\sigma$-closure-preserving open family of $Z$, which forms a local base of each point of $p(Y)$ in $Z$. Since there exists a $\sigma$-closure-preserving open family of $Z$ which forms a local base of each point of $Z-p(Y)$ in $Z, Z$ is shown to be an $M_{1}$-space.

Lemma 2. Let $Z=X \cup Y$ be a stratifiable space, where $X \cap Y=\varnothing, X$ is closed in $Z$ and $Y$ is $\sigma$-discrete. If $X \in \mathcal{P}$, then $Z \in \mathcal{P}$.

Proof. The proof is due to Gruenhage [5, Lemma 6.5]. Let $Y=\cup\left\{F_{n}: n \in N\right\}$, where each $F_{n}$ is closed discrete in $Y$ and $F_{m} \cap F_{n}=\varnothing$ if $m \neq n$. For each $x \in Y$ let $n(x)$ be the integer such that $x \in F_{n(x)}$. Let $H$ be an arbitrary closed subset of $Z$. Since $X \in \mathcal{P}, X \cap H$ has a closure-preserving open neighborhood base $\mathscr{Q}$. Take $U \in \mathcal{Q}$. Since $Y \cup U$ is stratifiable, the closed subset $H \cup U$ has a closure-preserving neighborhood base $\mathscr{B}$ in $Y \cup U$, which consists of closed neighborhoods in $Y \cup U$. Inductively, define, for each $x \in Y$, an open neighborhood $U(x)$ of $x$ in $Y$ such that

(i) $\left\{U(x): x \in F_{n}\right\}$ is a discrete, closed and open family of $Y$,

(ii)

$$
\begin{aligned}
U(x) \subset & D\left(\{x\}, \cup\left\{F_{i}: i<n(x)\right\} \cup(\cup\{B \in \mathscr{B}: x \notin B\})\right) \\
& \cap \cap\{U(y): x \in U(y), n(y)<n(x)\} .
\end{aligned}
$$

Then $\{U(x): x \in Y\}$ has the following properties:

(1) $y \in U(x)$ implies $U(y) \subset U(x)$,

(2) if $H$ is closed in $Y$, then $\cup\{U(y): y \in H\}$ is closed and open in $Y$, and

(3) $x \notin B \in \mathscr{B}$ implies $U(x) \cap B=\varnothing$.

For every open set $V$ of $Z$ satisfying $V \cap X=U$ and $H \subset V \subset U^{\prime}$, where $U^{\prime}$ is the special extension of an open set $H \cup U$ in the subspace $H \cup X$ to $Z$, define $W(V)=V-U\{U(x): x \in Y-V\}$. Set $\mathscr{W}(U)=\{W(V): V$ are the above open 


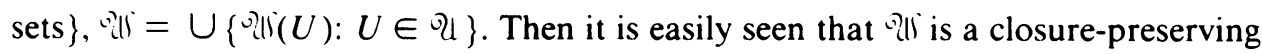
open neighborhood base of $H$ in $Z$.

THEOREM 3. If $X$ is a space such that each closed subspace of $X$ belongs to $\mathrm{PP}$, then the closed image of $X$ belongs to $\mathrm{p}$.

Proof. Let $f: X \rightarrow Y$ be a closed mapping. By [5, Lemma 6.1] there exists a closed subset $X_{0}$ of $X$ such that $f \mid X_{0}: X_{0} \rightarrow f\left(X_{0}\right)$ is irreducible and $Y-f\left(X_{0}\right)$ is open and

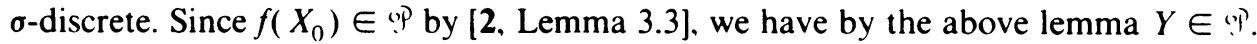

THEOREM 4. Let $X$ be a space such that, for each metrizable space $Y$, every closed subspace of $X \times Y$ is an $M_{1}$-space. Then $X \in$ ??

Proof. Let $F$ be a closed subset of $X$. Since $X$ is perfectly normal and submetrizable by the assumption, there exists a contraction $\rho$ of $X$ onto a metric space $\hat{X}$ such that $\rho(F)$ is closed in $\hat{X}$. There exists a perfect mapping $g$ of a zero-dimensional metric space $Y$ onto $\hat{X}$. Let $Z^{\prime}$ be the subspace of $X \times Y$ defined by

$$
Z^{\prime}=\{(x, y) \in X \times Y: \rho(x)=g(y)\} .
$$

Let $f^{\prime}, \sigma$ be the restrictions to $Z^{\prime}$ of the projections onto $X, Y$. respectively. By the argument of [11, Lemma 5.13. p. 293] $f^{\prime}$ is a perfect mapping of $Z^{\prime}$ onto $X$ and $\sigma$ is a contraction onto $Y$. By [11, Proposition 2.5, p. 219] there exists a closed subspace $Z$ of $Z^{\prime}$ such that $f=f^{\prime} \mid Z$ is a perfect and irreducible mapping of $Z$ onto $X$. Observe that $\sigma\left(f^{\prime-1}(F)\right)$ is closed in $Y$. Therefore $f^{-1}(F)$ has the form

$$
f^{-1}(F)=\bigcap\left\{G_{n}: n \in N\right\}, \quad G_{1}=Z, G_{n+1} \subset G_{n}, n \in N .
$$

where each $G_{n}$ is a closed and open set of $Z$. By the assumption, there exists a base $\because h=\cup\left\{\varphi_{i}: i \in N\right\}$ for $Z$, where each $\mathscr{B}_{i}$ is closure-preserving in $Z$. Set $B_{i}=B \cap G_{i}$, $B \in \bigcap_{i}, i \in N$. Let $\left\{\delta_{\beta}: \beta \in \Gamma\right\}$ be the totality of subcollections of $\varphi_{i}$. Set

$$
\begin{gathered}
V_{\beta i}=\bigcup\left\{B_{i}: B \in \mathcal{S}_{\beta} \cap \mathscr{B}_{i}\right\}, \quad V_{\beta}=\bigcup\left\{V_{\beta i}: i \in N\right\}, \\
B_{0}=\left\{\beta \in \Gamma: V_{\beta} \text { is an open set such that } f^{-1}(F) \subset V_{\beta}\right\}, \\
U_{\beta}=X-f\left(Z-V_{\beta}\right), \quad \beta \in B_{0}, \\
\mathscr{U}=\left\{U_{\beta}: \beta \in B_{0}\right\} .
\end{gathered}
$$

By a routine check, it is shown that $\left\{V_{\beta}: \beta \in B_{0}\right\}$ is a closure-preserving open neighborhood base of $f^{-1}(F)$ in $Z$. Since $f$ is closed and irreducible, $Q d$ is a closure-preserving open neighborhood base of $F$ in $X$ by [2, Lemma 3.3]. This completes the proof.

The author does not know the following: (1) Does every closed subspace of a space $\in \mathscr{P}$ belong to $\mathcal{P}$ ? (2) If $X, Y \in \mathscr{P}$, does $X \times Y \in \mathcal{P}$ ?

Finally, the author expresses his thanks for the referee's valuable suggestions.

\section{REFERENCES}

1. C. R. Borges, On stratifiable spaces, Pacific J. Math. 17 (1966), 1-16.

2. C. R. Borges and D. J. Lutzer. Characterization and mappings of $M_{1}$-spaces, Topology Conf. (Virginia Polytech. Inst. and State Univ., 1973), pp. 34-40.

3. J. G. Ceder, Some generalizations of metric spaces, Pacific J. Math. 11 (1961), 105-125.

4. G. Gruenhage. Stratifiable spaces are $M_{2}$, Topology Proc. 1 (1976), 221-226. 
5. On the $M_{3} \Rightarrow M_{1}$ question. Topology Proc. 5 (1980), 77-104.

6. R. W. Heath and H. J. K. Junnila, Stratifiable spaces as subspaces and continuous images of $M_{1}$-spaces. Proc. Amer. Math. Soc. 83 (1981), 146-148.

7. R. W. Heath, D. J. Lutzer and P. L. Zenor, Monotonically normal spaces, Trans. Amer. Math. Soc. 178 (1973), $481-493$.

8. H. J. K. Junnila, Neighhornets, Pacific J. Math. 76 (1968), 83-103.

9. K. Nagami, The equality of dimensions, Fund. Math. 106 (1980), 239-246.

10. J. Nagata, On Hyman's M-spaces, Topology Conf. (Virginia Polytech. Inst. and State Univ., 1973), pp. $198-208$.

I1. A. R. Pears, Dimension theory of general spaces, Cambridge Univ. Press, London and New York, 1975

Department of Mathematics, Joetsu University of Education, Joetsu, Niigata 943, Japan 